\title{
Schizophrenia and risk of dementia: a meta-analysis study
}

This article was published in the following Dove Press journal:

Neuropsychiatric Disease and Treatment

\section{Laisheng Cai \\ Jingwei Huang}

Department of Neurology, The First Affiliated Hospital of Nanchang University, Jiangxi, China
Correspondence: Jingwei Huang Department of Neurology,

The First Affiliated Hospital of Nanchang University, No 17 Yongwaizheng Street,

Donghu District, Jiangxi, China

Tel +86 I 3907083792

Email huangjw67@sohu.com
Background: Evidence suggests that schizophrenia may be associated with an increased risk of dementia, but results from prior studies have been inconsistent. This study aimed to estimate the relationship between schizophrenia and incident dementia using a quantitative meta-analysis. Methods: Several databases were used to gather relevant information, including PubMed, Embase, and Web of Science, with the publication date of articles limited up to December 23, 2017. All studies reported a multivariate-adjusted estimate, represented as relative risk (RR) with 95\% confidence intervals (CIs), for the association between schizophrenia and risk of dementia incidence. Pooled RRs were calculated using a random-effects model.

Results: Six studies met our inclusion criteria for this meta-analysis, which included 206,694 cases of dementia and 5,063,316 participants. All individuals were without dementia at baseline. Overall, the quantitative meta-analysis suggested that subjects with schizophrenia were associated with a significantly greater risk of dementia incidence (RR 2.29; 95\% CI 1.35-3.88) than those without.

Conclusion: The results of this meta-analysis indicate that individuals with schizophrenia may have an increased risk for the development of dementia. Future studies should explore whether schizophrenia is a modifiable risk factor for dementia.

Keywords: schizophrenia, dementia, meta-analysis

\section{Introduction}

Dementia is one of the most common and most devastating diseases of late life, and is a burdensome health condition worldwide. ${ }^{1}$ It is characterized by a progressive loss of cognitive and functional abilities related to behavioral disturbances, which consequentially leads to debility and death. ${ }^{2,3}$ Recent estimates suggest that approximately 44 million people currently suffer from dementia, with each year seeing an increase of 7.7 million new cases. ${ }^{4}$ Based on a shifting demographic and associated epidemiological profile worldwide, the number of people affected is predicted to double every 20 years and is creating a substantial financial, social, and public health burden, particularly in resource-poor countries. ${ }^{4,5}$

Schizophrenia is a brain disorder with symptoms of hallucinations and delusions, ${ }^{6}$ which is a chronic mental illness with a lifetime prevalence of nearly $1 \%$ in the general population. ${ }^{7}$ The disorder is related to disturbances in communication, perception, and thought processes, as well as abnormalities in behavior. ${ }^{8}$ Social disability and substantial functional impairment are common results of these disturbances. ${ }^{9}$ Diminished cognition is a basic feature of schizophrenia. ${ }^{10-14}$

Some studies have made an effort to evaluate the hypothesis of a shared etiology between schizophrenia and dementia by using mental disorders as determining predispositions for dementia. ${ }^{15}$ Longitudinal studies have confirmed the relationship between 
schizophrenia and dementia risk. ${ }^{16-18}$ A number of studies have found a significant cognitive decline over time in people with schizophrenia. ${ }^{16,19,20}$ However, some results suggested that the course of schizophrenia did not lead to dementia. ${ }^{21-25}$ Therefore, the relation between schizophrenia and the risk of subsequent dementia remains controversial.

Whether schizophrenia independently increases dementia incidence, or whether this correlation is confounded by traditional dementia predispositions, is unclear as previous studies have shown inconsistent results. ${ }^{26}$ Moreover, the past literature contained no adequate evidence on subgroups, such as study design (prospective vs retrospective), geographical area (Europe vs non-Europe), duration of follow-up ( $<10$ years vs $\geq 10$ years), quality (Newcastle-Ottawa Scale [NOS] score 8 vs 9 ), and age ( $<65$ years vs $\geq 65$ years). Thus, to obtain a more comprehensive estimate of the putative influence of schizophrenia on dementia, we performed a meta-analysis of studies. ${ }^{27}$

\section{Methods}

Recommended guidelines from the Preferred Reporting Items for Systematic Reviews and Meta-Analyses (PRISMA) statement were followed in the production of this metaanalysis. $^{28}$

\section{Literature search}

Two researchers performed a systematic review of peerreviewed papers from the databases of PubMed, Embase, and Web of Science, with the date restricted to articles published up to December 23, 2017. The database search keywords were: "Schizophrenias" OR "Schizophrenic Disorders" OR "Disorder, Schizophrenic" OR "Disorders, Schizophrenic" OR “Schizophrenic Disorder” OR “Dementia Praecox” AND "Dementias" OR “Amentia" OR “Amentias" OR "Senile Paranoid Dementia” OR "Dementias, Senile Paranoid" OR "Paranoid Dementia, Senile" OR "Paranoid Dementias, Senile" OR "Senile Paranoid Dementias" OR "Familial Dementia" OR "Dementia, Familial” OR "Dementias, Familial" OR "Familial Dementias" AND "prospective studies" OR "cohort studies" OR "longitudinal studies" OR "follow-up studies". We used Medical Subject Heading (MeSH) terms to retrieve literature in PubMed, and Emtree terms were used in Embase. The article search was limited to those published in the English language. In addition, we searched the reference lists of the included studies. The literature search, title/abstract screening, final decision on eligibility after full-text review, and data extraction were independently performed by two investigators.

\section{Study selection}

The following inclusion criteria were adopted: (1) the study of patients had a community-based or population-based design (prospective or retrospective); (2) the original article was not found in reviews, posters, or abstracts; (3) the exposure was schizophrenia; (4) there was at least a 12-month period of follow-up; and (5) the study reported quantitative estimates of the multivariate-adjusted relative risks (RRs) and $95 \%$ confidence intervals (CIs) for dementia related to schizophrenia; hazard ratios and odds ratios were considered equivalent to RRs. Exclusion criteria were as follows: (1) the study design method was cross-sectional; (2) animal or cell-line studies, commentaries, meta-analyses, case reports or series, reviews, meetings, and editorials or manuscripts unrelated to the research topic; (3) studies with shorter than a 12-month period of follow-up; and (4) studies with insufficient data to estimate adjusted RRs and 95\% CIs. We used EndNote to remove duplicate data. Titles and abstracts were screened for eligible studies and the full text was subsequently reviewed for potential qualifying studies. If multiple studies derived from the same research center, the authors were contacted to exclude overlapping samples.

\section{Data abstraction and quality assessment}

The two authors (LSC and JWH) independently extracted the data. If there was disagreement, a colleague (Liang Gao) was consulted to resolve the disagreement. The following data were extracted from each study: the first author's family name, year of publication, study design, country, location, number of participants, number of dementia cases, sex, mean age, follow-up time, assessment of schizophrenia and dementia, adjusted covariates, and study quality. The NOS criteria were used to evaluate the quality of the included original articles. ${ }^{29}$ The quality of studies was evaluated in the following three major components: selection of the study group (score $0-4$ ), quality of the adjustment for confounding (score 0-2), and assessment of outcome or exposure in the cohorts (score 0-3) (a total score of 0 denoted noncompliance with any criteria; 9 denoted complete compliance with all criteria). Studies were graded on a high to low point scheme, with a good quality score if they met $7-8$ points, fair if they met 4-6 points, and poor if they met 4 points.

\section{Statistical analysis}

All statistical analyses were performed using Stata software (version 12.0; StataCorp, College Station, TX, USA). The data analysis used multivariate adjusted RRs and 95\% CIs. We converted these values in every study by using their 
natural logarithms, and the SEs were calculated from these logarithmic numbers and their corresponding $95 \%$ CIs. ${ }^{30}$ The outcome was pooled using the random-effects model. ${ }^{31}$ $P$-values were two sided and $P<0.05$ was considered statistically significant.

\section{Results}

\section{Search results}

The initial search identified 127 records from PubMed, 51 records from Embase, 559 records from the Web of Science, and one record from other sources. After removing duplicate papers, 649 studies were identified in total. Scanning of titles and abstracts resulted in the identification of 38 articles for full-text scrutiny. In total, 32 studies were excluded: 25 lacked necessary data, ${ }^{10-14,16-20,32-46}$ one was a review, ${ }^{47}$ five did not report dementia outcome, ${ }^{21-25}$ and one was a sample duplication. ${ }^{15}$ Finally, there were six papers including 206,694 cases of dementia and 5,063,316 participants in the meta-analysis $^{48-53}$ (Figure 1).

\section{Study characteristics}

The general characteristics of the selected studies and participants included in the present meta-analysis are listed in Table 1. Among the six studies, four were conducted primarily in European countries, ${ }^{48,49,51,53}$ one was from an Asian country, ${ }^{50}$ and one was from Oceania. ${ }^{52}$ The follow-up duration ranged from 3 to 20 years; in three studies follow-up was $<10$ years $^{4,50,53}$ and in three studies $>10$ years. ${ }^{49,51,52}$ Four studies were prospective ${ }^{49-51,53}$ and two studies retrospective. ${ }^{48,52}$ All studies did not have sex-specific data, only two studies have reported the RR of men and women, respectively. ${ }^{50,51}$ All studies provided adjusted risk estimates. The overall assessment of studies was good (range 8-9 points). Dementia was present in schizophrenia patients of all ages. For age-group analysis, patients were divided into two categories $\left(<65\right.$ and $\geq 65$ years). ${ }^{50,51}$

\section{Quality assessment}

In accordance with NOS criteria, ${ }^{29}$ risk assessment for bias was carried out in the six included studies (Table 2). The results indicated that the methodological quality of the studies was satisfactory (range $8-9$ points).

\section{Schizophrenia and dementia incidence}

The multivariable adjusted RRs of dementia incidence in respect of schizophrenia from a single study and the

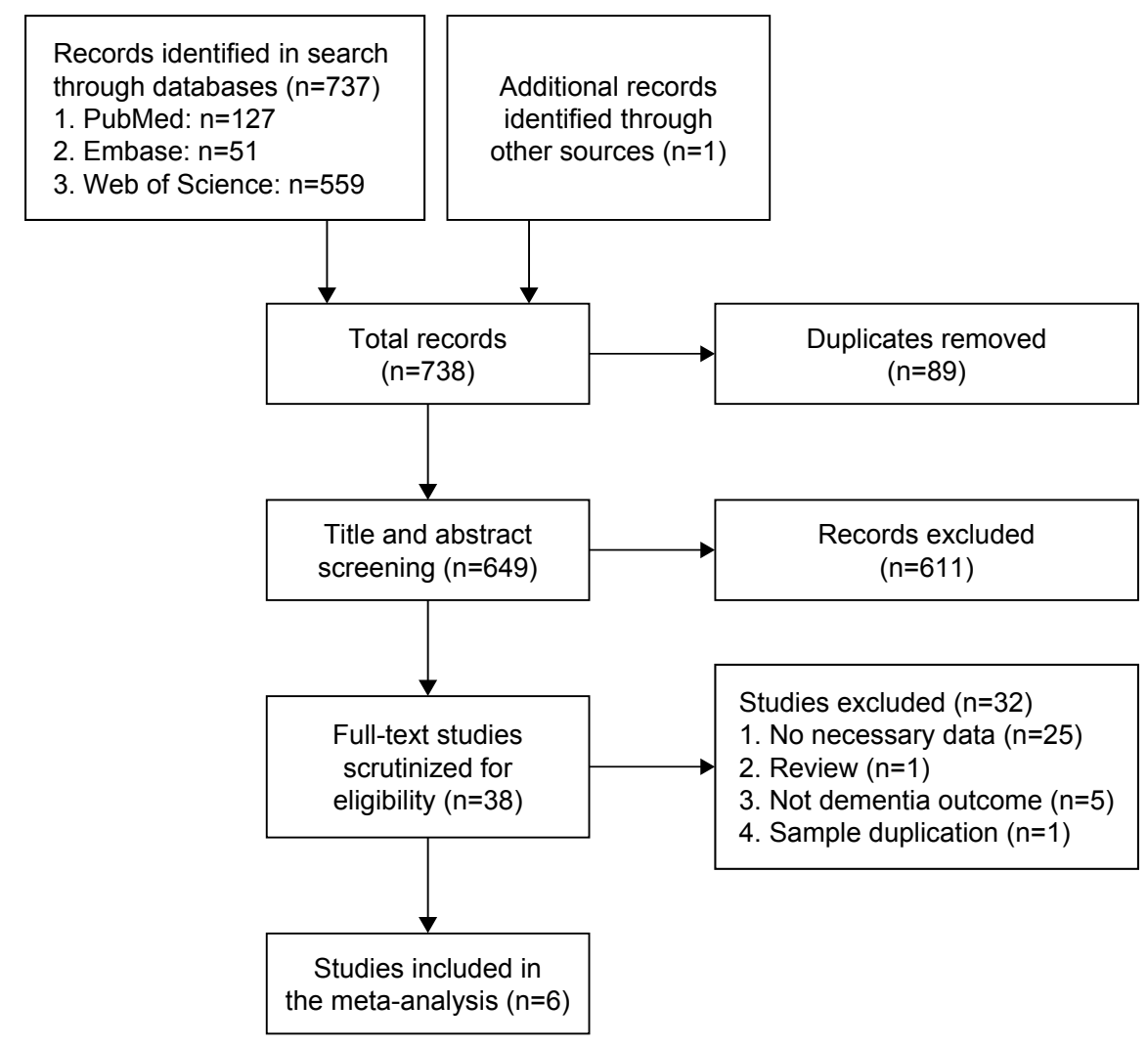

Figure I Flowchart depicting the literature search and study selection.

Notes: After removal of duplicates, reviews, and quality control, six papers were suitable for analysis. 


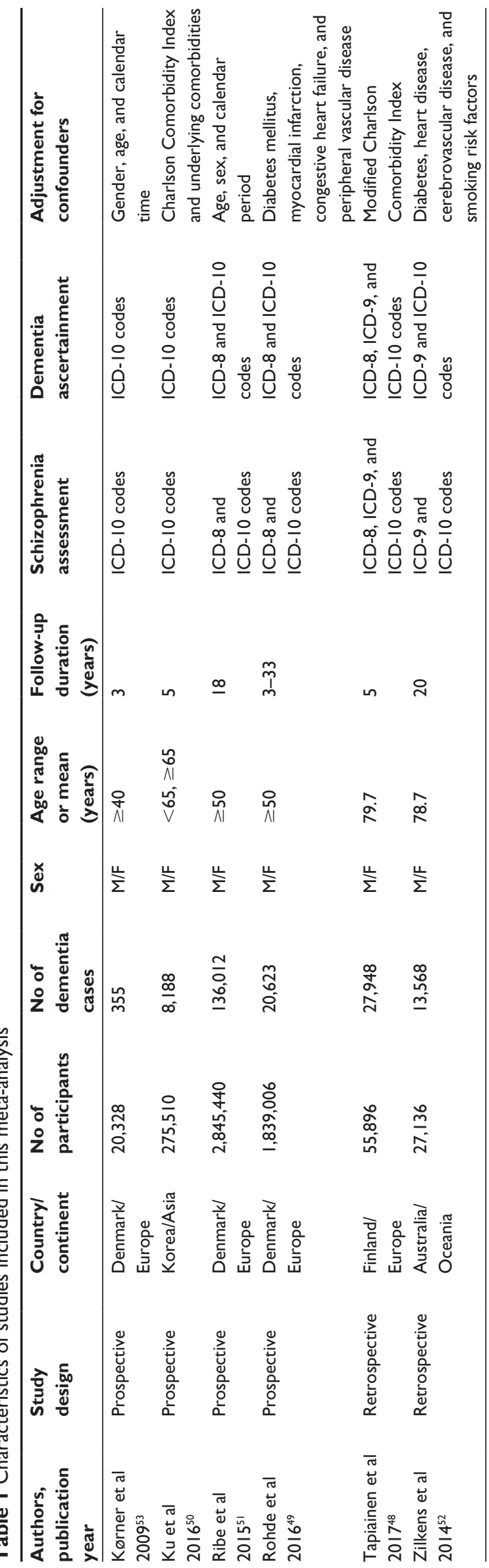

Table 2 Quality scores of included studies

\begin{tabular}{lllll}
\hline Study & Selection & Comparability & $\begin{array}{l}\text { Outcome/ } \\
\text { exposure }\end{array}$ & Total \\
\hline Kørner et al 200953 & 4 & I & 3 & 8 \\
Ku et al 201650 & 4 & 2 & 3 & 9 \\
Ribe et al 2015 51 & 4 & 2 & 3 & 9 \\
Rohde et al 201649 & 4 & I & 3 & 8 \\
Tapiainen et al 201748 & 4 & 2 & 3 & 9 \\
Zilkens et al 201452 & 4 & 2 & 3 & 9 \\
\hline
\end{tabular}

combined RR are presented in Figure 2. In Figure 2, all studies reported data on both men and women. The meta-analysis demonstrated that patients with schizophrenia, compared with non-schizophrenia subjects, had a significantly higher risk of developing dementia, based on the six studies ${ }^{48-53}$ (combined RR 2.29; 95\% CI 1.35-3.88). Heterogeneity was significantly observed $\left(P=0.000 ; I^{2}=98.9 \%\right)$. The forest plots of multivariable RRs and $95 \%$ CIs for dementia incidence and schizophrenia are shown in Figure 2.

\section{Subgroup analysis}

To further elucidate the role of schizophrenia in stroke, we next performed a subgroup analysis based on the six studies. ${ }^{48-53}$ The pooled RRs for dementia stratified by study design, geographical area, duration of follow-up, study quality, and age range or mean age of participants are shown in Figure 3. In Figure 3, the estimated RR for studies from Europe was 1.66 (95\% CI 1.03-2.66) and from non-European countries was 4.70 (95\% CI 4.37-5.06), and the pooled estimate of multivariate RRs of dementia incidence was 3.36 (95\% CI 1.24-9.13) among women and 3.09 (95\% CI 1.85-5.13) among men. We found significant associations in the overall subgroup meta-analysis by age. Furthermore, increases in dementia events were found in the subgroup of study design (prospective study: RR $2.52,95 \%$ CI 1.47-4.34). In addition, we found significant associations in the overall subgroup meta-analysis by duration of follow-up ( $>10$ years) and study quality ( 8 points).

\section{Sensitivity analysis}

Sensitivity analysis was performed by omitting one study at a time and reassessing the summary RR for the remaining studies. This demonstrated that no single study significantly influenced the statistically significant differences in dementia incidence, and indicated that the results of the present metaanalysis were firm (Figure 4).

\section{Discussion}

The meta-analysis of six studies involving 5,063,316 participants and 206,694 cases showed a significant positive 


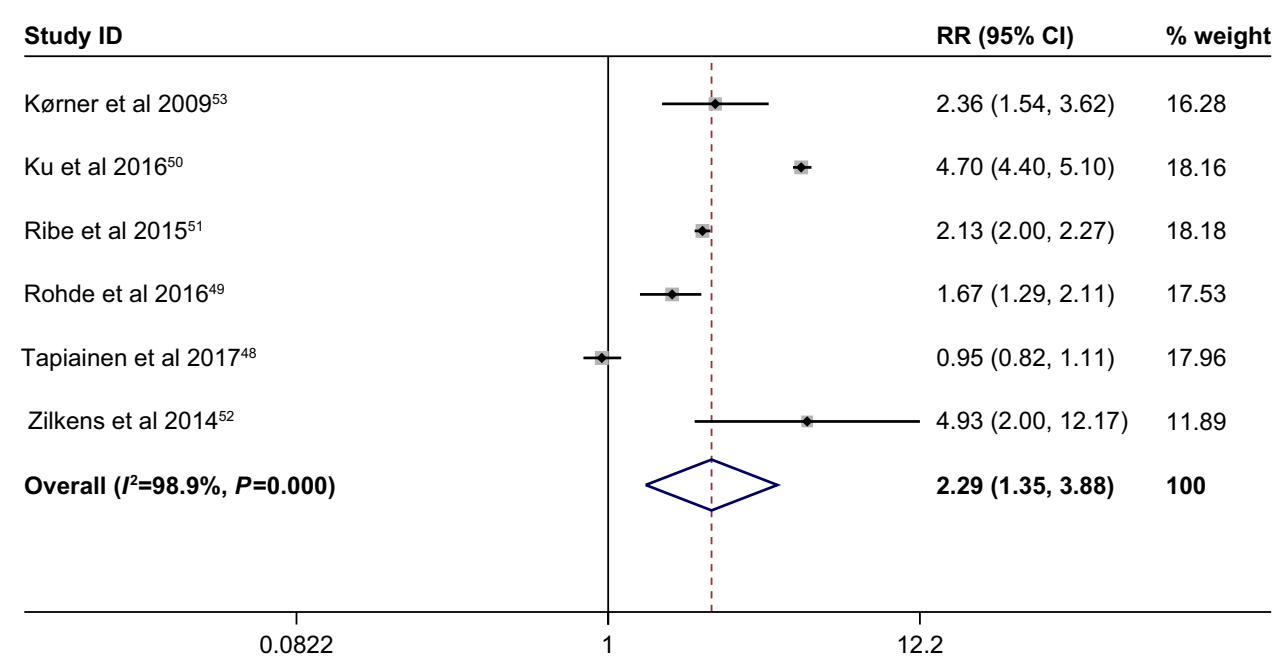

Figure 2 Meta-analysis of the association between schizophrenia and dementia incidence. The random-effects model was used. There was a highly significant difference between the two groups $(P=0.002)$.

Note: Weights are from random effects analysis.

Abbreviation: $\mathrm{RR}$, relative risk.

relationship between schizophrenia and risk of dementia. These results implied that subjects with schizophrenia may have a higher prevalence of dementia than those without schizophrenia.
To a certain degree, the RR of dementia was higher among women than men. Some studies have shown that the higher dementia incidence in women is due to various mechanisms. First, women often end up living alone without a spouse when

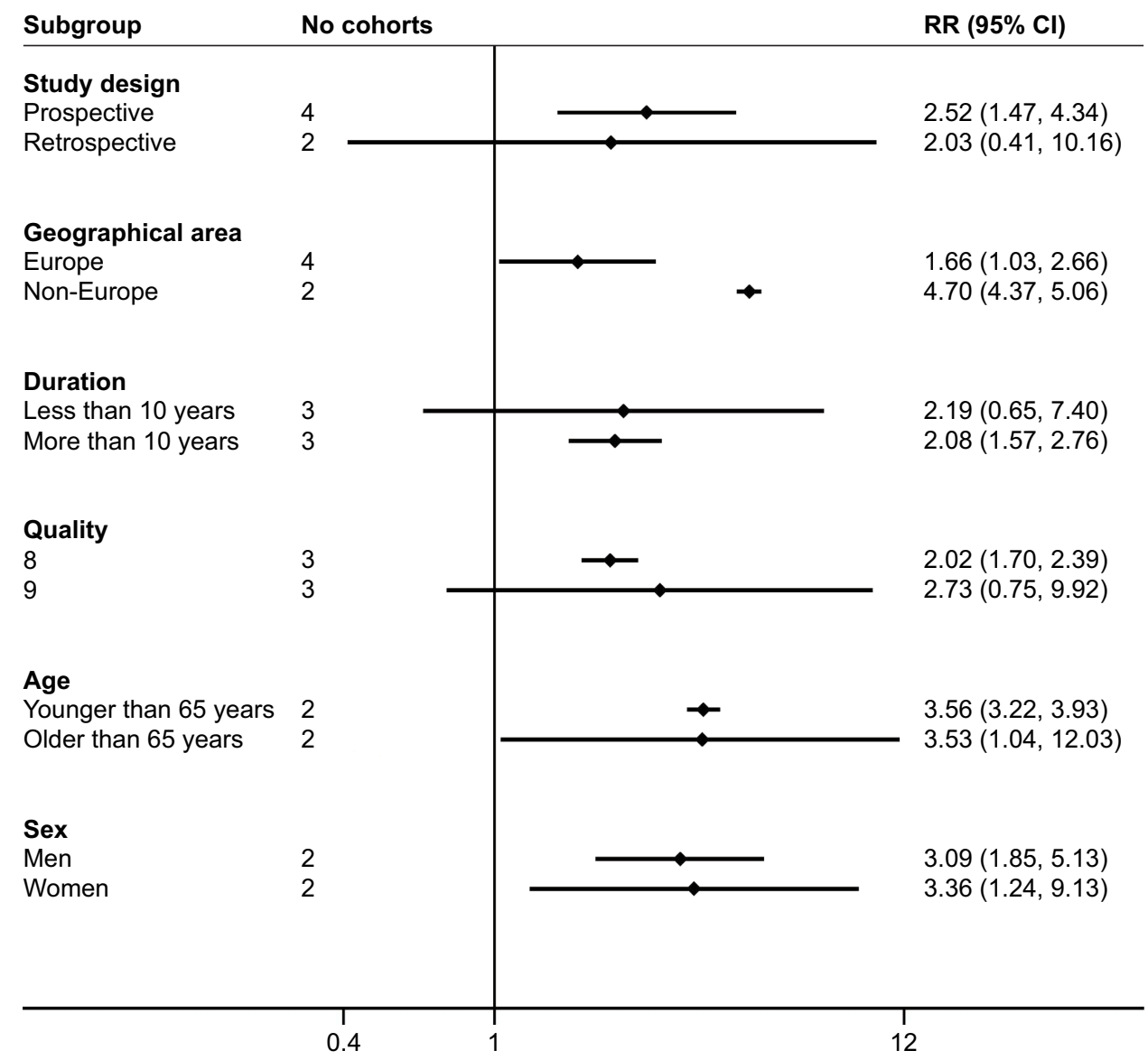

Figure 3 Analyses of subgroups relating schizophrenia to dementia. The random-effects model was applied. Abbreviation: RR, relative risk. 
Meta-analysis estimates, given named study is omitted

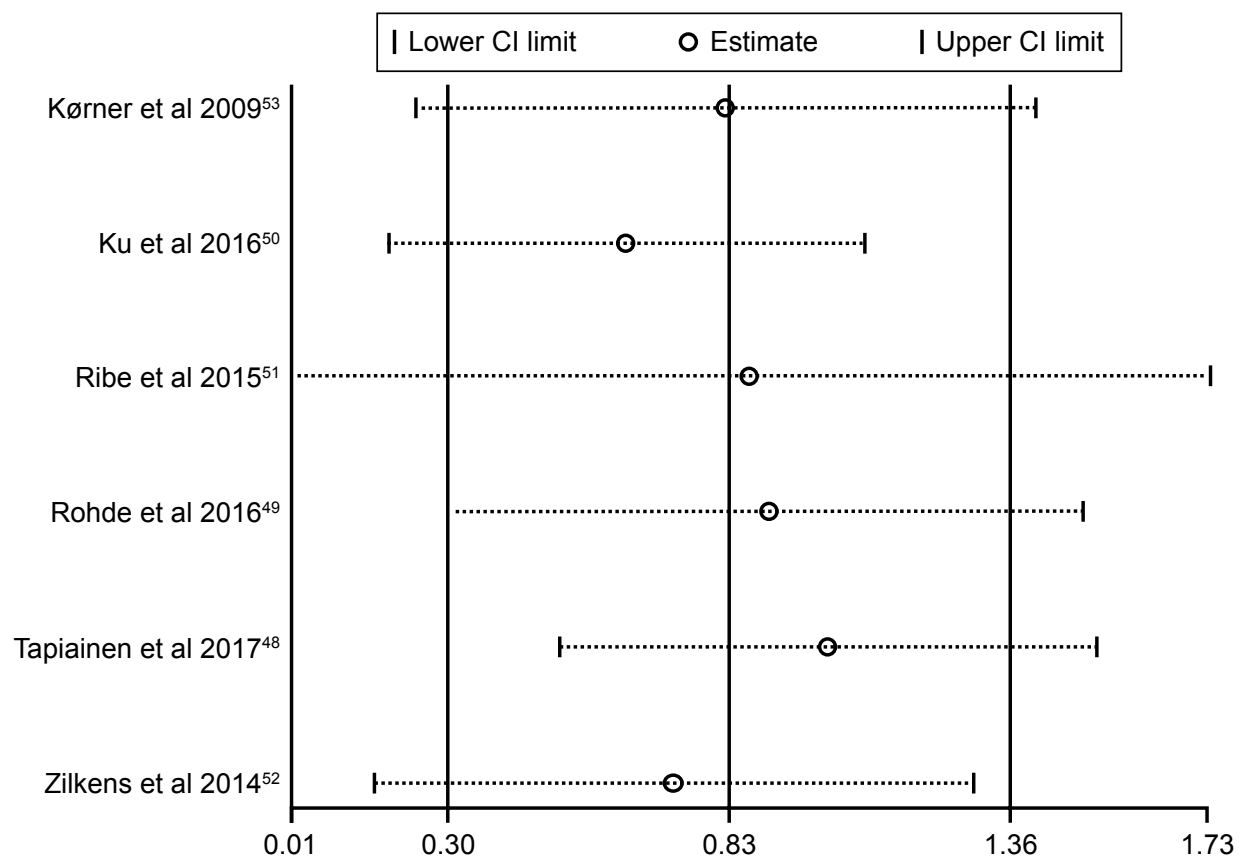

Figure 4 Sensitivity analyses results when the given named study was omitted.

they reach the age of 80 or 85 because women often marry older men, and men live shorter lives on average. Loneliness in old age is associated with a risk of dementia. ${ }^{54}$ Second, women have decreased estrogen levels after menopause, leading to some physiological changes, which also lead to changes in brain nerves and brain function. This change is expressed through decreased cognitive function, especially diminished short-term memory. ${ }^{55}$ Third, the most dominant genetic risk factor for dementia is the apolipoprotein E4 (APOE4) allele. Case-controlled studies suggest that the APOE4 link to dementia is stronger in women than in men. ${ }^{56}$ Fourth, women are prone to mood swings, insomnia, irritability, anxiety, and depression, which affect brain function to a certain extent and may hasten the progression of dementia.

Schizophrenia is a severe psychotic disorder characterized by a variety of signs and symptoms, including sensory hallucinations, delusions, apathy, lack of volition, disorganized language or behavior, and poverty of speech. ${ }^{14}$ Schizophrenia was historically regarded as "dementia praecox" because cognitive impairment is a common feature of schizophrenia. ${ }^{19}$ There is evidence that cognitive changes present early in the course of schizophrenia. ${ }^{57}$ Some studies suggest that cognitive decline occurs steadily throughout the course of the disease, as might occur in a progressive, neurodegenerative disorder. ${ }^{58,59}$ The cognitive and behavioral changes in schizophrenia are often severe enough to meet standard diagnostic criteria for dementia. ${ }^{25,46,53,60-62}$

Although the reason for the prevalence of dementia in people with schizophrenia is not well understood, several explanations are possible. First, schizophrenia patients could be a proxy variable for several chronic conditions, including cerebrovascular disease, ${ }^{63}$ substance abuse,${ }^{64}$ atrial fibrillation or flutter ${ }^{65,66}$ peripheral vascular disease, ${ }^{63,67}$ diabetes mellitus ${ }^{68}$ ischemic heart disease,,${ }^{63,67,69}$ and congestive heart failure, ${ }^{63,65}$ all of which are well-established risk factors for dementia. Second, the mechanism of schizophrenia is linked to cognitive and memory impairment, which has led to progressive brain dysfunction. ${ }^{50}$ Third, a hypothesis has been suggested that the higher risk of dementia could reflect a common underlying etiology for schizophrenia and dementia. ${ }^{70}$ Finally, the higher risk might be explained as being due to antipsychotics $^{50}$ and adverse health risk factors (such as alcohol consumption, tobacco smoking, and low physical activity) for individuals with schizophrenia. The cumulative use of antipsychotics for schizophrenia patients was related to cognitive decline in an observational follow-up study. ${ }^{71}$ Moreover, there is evidence that the excess risk of dementia in subjects with schizophrenia may be explained by adverse health risk factors. ${ }^{15}$ Thus, there are many potential reasons why people with schizophrenia may have progressive cognitive impairment during the course of the disease. ${ }^{19}$ 
Heterogeneity may come from several sources; therefore, subgroup analysis and sensitivity analysis were used to explore the possible confounders that explained the high level of between-study heterogeneity. The subgroup analysis suggested that the associations were still significant in different subgroups except in the retrospective study, with less than 10 years' duration and 9 points of quality (Figure 3 ). Thus, study design, follow-up duration, and study quality partly accounted for the significant heterogeneity. The heterogeneity may also be attributable to differences in environmental factors, physical condition, unhealthy lifestyles, selection bias, and how the studies were conducted. However, heterogeneity between studies was found in dementia incidence, which had little influence in the sensitivity analysis (Figure 4). Although the existence of confounding factors could not be completely eliminated in the study populations, the recruitment of participants in the six eligible studies met the strict inclusion criteria. In summary, there was significant heterogeneity among individual studies, but the outcomes of our study were reliable.

The observational studies in this meta-analysis contained a number of limitations. First, it would be interesting to identify whether the schizophrenia-dementia association differed by schizophrenia subtype (eg, paranoid vs hebephrenic) and dementia subtype (eg, Alzheimer's disease and vascular dementia), but few data were available for a stratified analysis. Second, we included only six studies, some of which had a relatively small sample size. The limited number of included studies meant that we were only able to include a small number of moderators in the subgroup analysis. The small number of studies providing data on patient-related moderators made it difficult to undertake further analysis (such as meta-regression and checks for publication bias). Third, language bias and selection bias might be unavoidable. We performed searches on three major electronic databases with the limitation of English language-based articles. Thus, some literature published in Chinese or other non-English languages may have been missed. Furthermore, the majority of studies included were from Western countries, while only one study was from Asia and one from Oceania, and there were no relevant reports from any African populations. Fourth, it is well established that the early mortality rate of schizophrenia patients is higher than in the general population; thus, these patients may have died before developing dementia. This possibility may make the result a conservative one. Finally, confounding from other risk factors (eg, poor health care, unhealthy lifestyles, low education, antischizophrenia or antipsychotic drug effects) may have affected the significant relation between schizophrenia and dementia risk. These limits highlight the need for continued investigations into schizophrenia in dementia.

\section{Conclusion}

The results of this meta-analysis show that schizophrenia is significantly associated with an increased risk of dementia. Our findings suggest that individuals with schizophrenia should be provided with efficient prevention strategies against dementia or that medical treatments for schizophrenia that may decrease the risk of dementia should be chosen. Further studies, especially randomized controlled studies of agents that lower or prevent elevated schizophrenia, should explore whether or not schizophrenia is a potentially modifiable risk factor for dementia.

\section{Acknowledgment}

We would like to thank Liang Gao for providing help with the meta-analysis.

\section{Disclosure}

The authors report no conflicts of interest in this work.

\section{References}

1. Ferri CP, Prince M, Brayne C, et al. Global prevalence of dementia: a Delphi consensus study. Lancet. 2005;366(9503):2112-2117.

2. van der Flier WM, Scheltens P. Epidemiology and risk factors of dementia. J Neurol Neurosurg Psychiatry. 2005;76(Suppl 5):v2-v7.

3. Nilsson K, Gustafson L, Hultberg B. Survival in a large elderly population of patients with dementia and other forms of psychogeriatric diseases. Dement Geriatr Cogn Disord. 2011;32(5):342-350.

4. Wortmann M. Dementia: a global health priority - highlights from an ADI and World Health Organization report. Alzheimers Res Ther. 2012; 4(5):40.

5. Prince M, Bryce R, Albanese E, Wimo A, Ribeiro W, Ferri CP. The global prevalence of dementia: A systematic review and metaanalysis. Alzheimers Dement. 2013;9(1):63.e2-75.e2.

6. Walker E, Kestler L, Bollini A, Hochman KM. Schizophrenia: etiology and course. Апnи Rev Psychol. 2004;55(1):401-430.

7. Freedman R. Schizophrenia. N Engl J Med. 2003;349(18): $1738-1749$.

8. Flaum M, Schultz SK. The core symptoms of schizophrenia. Ann Med. 1996;28(6):525-531.

9. Viertiö S, Tuulio-Henriksson A, Perälä J, et al. Activities of daily living, social functioning and their determinants in persons with psychotic disorder. Eur Psychiatry. 2012;27(6):409-415.

10. Harvey PD, Leff J, Trieman N, Anderson J, Davidson M. Cognitive impairment in geriatric chronic schizophrenic patients: a cross-national study in New York and London. Int J Geriatr Psychiatry. 1997; 12(10):1001-1007.

11. Harvey PD, Lombardi J, Leibman M, et al. Cognitive impairment and negative symptoms in geriatric chronic schizophrenic patients: a follow-up study. Schizophr Res. 1996;22(3):223-231.

12. Harvey PD. Cognitive impairment in elderly patients with schizophrenia: age related changes. Int J Geriatr Psychiatry. 2001;16 Suppl 1(S1): S78-S85. 
13. Harvey PD, Reichenberg A, Bowie CR. Cognition and aging in psychopathology: focus on schizophrenia and depression. Annu Rev Clin Psychol. 2006;2(1):389-409.

14. Man DW. Cognitive functions among Hong Kong Chinese people with schizophrenia. Int J Rehabil Res. 2006;29(3):261-265.

15. Rohde C, Agerbo E, Nielsen PR. Does Schizophrenia in Offspring Increase the Risk of Developing Alzheimer's Dementia. Dement Geriatr Cogn Dis Extra. 2016;6(2):361-373.

16. Harvey PD, Silverman JM, Mohs RC, et al. Cognitive decline in late-life schizophrenia: a longitudinal study of geriatric chronically hospitalized patients. Biol Psychiatry. 1999;45(1):32-40.

17. Katagiri H, Okada G, Jitsuiki H, Kozuru T, Muraoka M, Yamawaki S. The longitudinal change of cognitive function of long-stay in patients with schizophrenia by the revised version of Hasegawa's Dementia Scale. International Journal of Neuropsychopharmacology. 2008;11: 165 .

18. Nayak Savla G, Moore DJ, Roesch SC, Heaton RK, Jeste DV, Palmer BW. An evaluation of longitudinal neurocognitive performance among middle-aged and older schizophrenia patients: Use of mixedmodel analyses. Schizophr Res. 2006;83(2-3):215-223.

19. Shah JN, Qureshi SU, Jawaid A, Schulz PE. Is there evidence for late cognitive decline in chronic schizophrenia? Psychiatr Q. 2012;83(2): $127-144$.

20. Friedman JI, Harvey PD, Coleman T, et al. Six-year follow-up study of cognitive and functional status across the lifespan in schizophrenia: a comparison with Alzheimer's disease and normal aging. Am J Psychiatry. 2001;158(9):1441-1448.

21. Harvey PD, White L, Parrella M, et al. The longitudinal stability of cognitive impairment in schizophrenia. Mini-mental state scores at oneand two-year follow-ups in geriatric in-patients. Br J Psychiatry. 1995; 166(5):630-633.

22. Hyde TM, Nawroz S, Goldberg TE, et al. Is there cognitive decline in schizophrenia? A cross-sectional study. Br J Psychiatry. 1994;164(4): 494-500.

23. Jellinger KA, Gabriel E. No increased incidence of Alzheimer's disease in elderly schizophrenics. Acta Neuropathol. 1999;97(2):165-169.

24. Laks J, Fontenelle LF, Chalita A, Mendlowicz MV. Absence of dementia in late-onset schizophrenia: a one year follow-up of a Brazilian case series. Arq Neuropsiquiatr. 2006;64(4):946-949.

25. Rabins PV, Lavrisha M. Long-term follow-up and phenomenologic differences distinguish among late-onset schizophrenia, late-life depression, and progressive dementia. Am J Geriatr Psychiatry. 2003;11(6):589-594.

26. Li M, Fan Y-L, Tang Z-Y, Cheng X-S. Schizophrenia and risk of stroke: A meta-analysis of cohort studies. Int J Cardiol. 2014;173(3):588-590.

27. Li M, Li K, Zhang X-W, Hou W-S, Tang Z-Y. Habitual snoring and risk of stroke: A meta-analysis of prospective studies. Int J Cardiol. 2015;185:46-49.

28. Moher D, Liberati A, Tetzlaff J, Altman DG. Preferred reporting items for systematic reviews and meta-analyses: The PRISMA statement. Int J Surg. 2010;8(5):336-341.

29. Stang A. Critical evaluation of the Newcastle-Ottawa scale for the assessment of the quality of nonrandomized studies in meta-analyses. Eur J Epidemiol. 2010;25(9):603-605.

30. Li M, Hou W, Zhang X, Hu L, Tang Z. Hyperuricemia and risk of stroke: A systematic review and meta-analysis of prospective studies. Atherosclerosis. 2014;232(2):265-270.

31. Dersimonian R, Laird N. Meta-analysis in clinical trials. Control Clin Trials. 1986;7(3):177-188.

32. Östling S, Pálsson SP, Skoog I. The incidence of first-onset psychotic symptoms and paranoid ideation in a representative population sample followed from age 70-90 years. Relation to mortality and later development of dementia. Int J Geriatr Psychiatry. 2007;22(6):520-528.

33. Kessing LV, Olsen EW, Mortensen PB, Andersen PK. Dementia in affective disorder: a case-register study. Acta Psychiatr Scand. 1999;100(3): 176-185.

34. Watson A, Joyce E. Cognitive reserve and neuropsychiatric disorders. Curr Opin Behav Sci. 2015;4:142-146.
35. Friedman JI, Harvey PD, Kemether E, Byne W, Davis KL. Cognitive and functional changes with aging in schizophrenia. Biol Psychiatry. 1999;46(7):921-928.

36. Chan H-M, Stolwyk R, Kelso W, et al. Comparing neurocognition in severe chronic schizophrenia and frontotemporal dementia. Aust NZJ Psychiatry. 2014;48(9):828-837.

37. Urfer-Parnas A, Mortensen EL, Parnas J. Core of schizophrenia: estrangement, dementia or neurocognitive disorder? Psychopathology. 2010; 43(5):300-311.

38. Song M-H, Hamada H, Mimura M. Semiological differences between late-life schizophrenia and senile dementia. Keio J Med. 2014;63(2): 34-38.

39. Seidman LJ, Mirsky AF. Evolving Notions of Schizophrenia as a Developmental Neurocognitive Disorder. J Int Neuropsychol Soc. 2017;23(9-10):881-892.

40. Schmid LA, Lässer MM, Herold C, Schröder J. Cognitive function of chronic schizophrenic patients in late life. Schizophr Bull. 2011;37: 252-253. Available from: http://dx.doi.org/10.1093/schbul/sbq173. Accessed August 07, 2018.

41. Schmid LA, Laesser MM, Schroeder J. Symptoms and cognition in geriatric schizophrenia. Fortschr Neurol Psychiatrie. 2011;79(5): 267-276.

42. Häfner H. Schizophrenia: still Kraepelin's dementia praecox? Epidemiol Psichiatr Soc. 2004;13(2):99-112.

43. Cohen CI, Murante T. A prospective analysis of the role of cognition in three models of aging and schizophrenia. Schizophr Res. 2018; 196:22-28.

44. Nicolas G, Beherec L, Hannequin D, et al. Dementia in middle-aged patients with schizophrenia. J Alzheimers Dis. 2014;39(4):809-822.

45. Girard C, Simard M, Noiseux R, et al. Late-onset-psychosis: cognition. Int Psychogeriatr. 2011;23(8):1301-1316.

46. Brodaty H, Sachdev P, Koschera A, Monk D, Cullen B. Long-term outcome of late-onset schizophrenia: 5-year follow-up study. $\mathrm{Br} J$ Psychiatry. 2003;183(3):213-219.

47. Harvey PD. Cognitive and functional impairments in elderly patients with schizophrenia: a review of the recent literature. Harv Rev Psychiatry. 2001;9(2):59-68.

48. Tapiainen V, Hartikainen S, Taipale H, Tiihonen J, Tolppanen A-M. Hospital-treated mental and behavioral disorders and risk of Alzheimer's disease: A nationwide nested case-control study. Eur Psychiatry. 2017;43:92-98.

49. Rohde C, Agerbo E, Nielsen PR. Spouse with schizophrenia and risk of dementia. Soc Psychiatry Psychiatr Epidemiol. 2016;51(12):1655-1658.

50. $\mathrm{Ku} \mathrm{H}$, Lee E-K, Lee K-U, Lee M-Y, Kwon J-W. Higher prevalence of dementia in patients with schizophrenia: A nationwide population-based study. Asia Pac Psychiatry. 2016;8(2):145-153.

51. Ribe AR, Laursen TM, Charles M, et al. Long-term Risk of Dementia in Persons With Schizophrenia: A Danish Population-Based Cohort Study. JAMA Psychiatry. 2015;72(11):1095-1101.

52. Zilkens R, Bruce D, Duke J, Spilsbury K, Semmens J. Severe psychiatric disorders in mid-life and risk of dementia in late-life (age 65-84 years): a population based case-control study. Curr Alzheimer Res. 2014; 11(7):681-693.

53. Kørner A, Lopez AG, Lauritzen L, Andersen PK, Kessing LV. Late and very-late first-contact schizophrenia and the risk of dementia-a nationwide register based study. Int J Geriatr Psychiatry. 2009;24(1): 61-67.

54. Wald C. Social networks: Better together. Nature. 2016;531(7592): S14-S15.

55. Fratiglioni L, Launer LJ, Andersen K, et al. Incidence of dementia and major subtypes in Europe: A collaborative study of population-based cohorts. Neurologic Diseases in the Elderly Research Group. Neurology. 2000;54(11 Suppl 5):S10-S15.

56. Altmann A, Tian L, Henderson VW, Greicius MD. Alzheimer's Disease Neuroimaging Initiative Investigators. Sex modifies the APOErelated risk of developing Alzheimer disease. Ann Neurol. 2014;75(4): 563-573. 
57. Asarnow RF. Neurocognitive impairments in schizophrenia: A piece of the epigenetic puzzle. Eur Child Adolesc Psychiatry. 1999;8(S1): S5-S8.

58. Lieberman JA. Is schizophrenia a neurodegenerative disorder? a clinical and neurobiological perspective. Biol Psychiatry. 1999;46(6): 729-739.

59. Goldberg TE, Hyde TM, Kleinman JE, Weinberger DR. Course of schizophrenia: neuropsychological evidence for a static encephalopathy. Schizophr Bull. 1993;19(4):797-804.

60. de Vries PJ, Honer WG, Kemp PM, Mckenna PJ. Dementia as a complication of schizophrenia. J Neurol Neurosurg Psychiatry. 2001; 70(5):588-596.

61. Banaschewski T, Schulz E, Martin M, Remschmidt H. Cognitive functions and psychopathological symptoms in early-onset schizophrenia. Eur Child Adolesc Psychiatry. 2000;9(1):11-20.

62. Ciompi L, Clemens S. Catamnestic long-term study on the course of life and aging of schizophrenics. Schizophr Bull. 1980;6(4):606-618.

63. Fan Z, Wu Y, Shen J, Ji T, Zhan R. Schizophrenia and the risk of cardiovascular diseases: A meta-analysis of thirteen cohort studies. J Psychiatr Res. 2013;47(11):1549-1556.

64. Nordentoft M, Mortensen PB, Pedersen CB. Absolute risk of suicide after first hospital contact in mental disorder. Arch Gen Psychiatry. 2011;68(10):1058-1064.
65. Rusanen M, Kivipelto M, Levälahti E, et al. Heart diseases and longterm risk of dementia and Alzheimer's disease: a population-based CAIDE study. J Alzheimers Dis. 2014;42(1):183-191.

66. Bresee LC, Majumdar SR, Patten SB, Johnson JA. Prevalence of cardiovascular risk factors and disease in people with schizophrenia: A population-based study. Schizophr Res. 2010;117(1):75-82.

67. Newman AB, Fitzpatrick AL, Lopez O, et al. Dementia and Alzheimer's disease incidence in relationship to cardiovascular disease in the Cardiovascular Health Study cohort. J Am Geriatr Soc. 2005;53(7): 1101-1107.

68. Dixon L, Weiden P, Delahanty J, et al. Prevalence and correlates of diabetes in national schizophrenia samples. Schizophr Bull. 2000; 26(4):903-912.

69. Laursen TM, Munk-Olsen T, Agerbo E, Gasse C, Mortensen PB. Somatic hospital contacts, invasive cardiac procedures, and mortality from heart disease in patients with severe mental disorder. Arch Gen Psychiatry. 2009;66(7):713-720.

70. Lyketsos CG, Peters ME. Dementia in Patients With Schizophrenia: Evidence for Heterogeneity. JAMA Psychiatry. 2015;72(11):1075-1076.

71. Husa AP, Rannikko I, Moilanen J, et al. Lifetime use of antipsychotic medication and its relation to change of verbal learning and memory in midlife schizophrenia - An observational 9-year follow-up study. Schizophr Res. 2014;158(1-3):134-141.
Neuropsychiatric Disease and Treatment

\section{Publish your work in this journal}

Neuropsychiatric Disease and Treatment is an international, peerreviewed journal of clinical therapeutics and pharmacology focusing on concise rapid reporting of clinical or pre-clinical studies on a range of neuropsychiatric and neurological disorders. This journal is indexed on PubMed Central, the 'PsycINFO' database and CAS,

\section{Dovepress}

and is the official journal of The International Neuropsychiatric Association (INA). The manuscript management system is completely online and includes a very quick and fair peer-review system, which is all easy to use. Visit http://www.dovepress.com/testimonials.php to read real quotes from published authors. 